\title{
Cirugía y traumatología bucomáxilofacial en la red hospitalaria chilena
}

\section{Maxilofacial surgery in the chilean healthcare network}

\author{
Bernardita Zúñiga1 ${ }^{1}$, Manuel González², Andrea González¹, Jorge Gamonal ${ }^{3}$
}

\author{
1. Universidad de Chile, Facultad de Odontologia. \\ Departamento de Cirugía y Traumatología Bucal y \\ Máxilofacial. \\ 2. Ingeniero Civil. Universidad de Chile. \\ 3. Laboratorio de Biología Periodontal, Centro de \\ Investigación y Vigilancia de las Enfermedades \\ Orales (CEVEO), Facultad de Odontología, \\ Universidad de Chile.
}

Correspondencia a: Bernardita Zúñiga Oliva. | +56 9 82323772. - +56229781841|

bzuniga@odontologia.uchile.cl

\section{RESUMEN}

Argumentando la existencia de listas de espera de cirugía bucal y máxilofacial, los Ministerios de Salud y Educación chilenos deciden crear un profesional de menores competencias que el cirujano bucal y máxilofacial existente, cuya formación dure un año y se desempeñe en la atención primaria de salud. Decretan la especialidad de Cirugía Bucal, comprometiendo la calidad y seguridad de la atención, exponiendo a los enfermos a eventos adversos de impredecibles desenlaces, afectando la calidad de vida de éstos o provocando eventualmente un desenlace fatal.

El objetivo del presente estudio es establecer el número de cirujanos bucales y máxilofaciales formados en el país, conocer cuantos se desempeñan en hospitales públicos y su distribución en los servicios de salud, así como también evaluar si se cumplen las condiciones de infraestructura necesarias para desarrollar la especialidad según la normativa vigente. Consideramos que éstos son datos fundamentales al momento de modificar políticas públicas.

La metodología propuesta es un estudio descriptivo de corte transversal, realizando una encuesta dirigida a los jefes de los servicios odontológicos de la red hospitalaria pública del país, destinada a obtener información del prestador individual e institucional en cada centro de la red.

Se concluye que existe una cantidad suficiente de especialistas formados y en vías de formación para atender las necesidades de la población. La evidencia muestra que faltan cargos e infraestructura, por lo que la medida adoptada por el MINSAL respecto a la creación de la especialidad de Cirugía Bucal obedece a un error diagnóstico desde el punto de vista del prestador individual e institucional, originado por la ausencia de un estudio técnico que evalúe la red hospitalaria del país.

\section{PALABRAS CLAVE}

Cirugía bucal y Máxilofacial, Calidad en atención de salud, Red Hospitalaria Pública.

Rev. Clin. Periodoncia Implantol. Rehabil. Oral Vol. 10(1); 57-63, 2017.

\section{ABSTRACT}

Arguing the existence of waiting lists in oral and maxillofacial surgery, the chilean Ministries of Health and Education decided to create a professional less skilled than the existing oral and maxilofacial surgeon, whose training lasts one year and works in primary care health. They established the specialty of Oral Surgery, compromising the quality and safety of care, exposing patients to adverse events with unpredictable outcomes, affecting their quality of life or eventually causing a fatal outcome.

At the time of this study there is no record of the number of oral and maxillofacial surgeons trained in our country, it is unknown how many are working in public hospitals and their distribution in health services, as well as whether there is the necessary infrastructure to develop the specialty under current regulations. We made a descriptive transversal study, conducting an interview aimed to obtain information from individual and institutional providers to know the reality of the specialty in the public health network.

It is concluded that there is a sufficient number of specialists trained and in training to meet the needs of the population. The evidence shows missing possitions and infrastructure, so that the action taken by the Health Ministry regarding the creation of the specialty of Oral Surgery is due to a misdiagnosis from the point of view of an individual and institutional provider, caused by the absence of a technical study to assess the country's hospital network.

\section{KEYWORDS}

Oral and Maxillofacial Surgery, Quality of Healthcare, Public Hospital.

Rev. Clin. Periodoncia Implantol. Rehabil. Oral Vol. 10(1); 57-63, 2017. 


\section{INTRODUCCIÓN}

La Constitución establece que la salud es un derecho ciudadano y con objeto de lograr que ésta sea otorgada con mayor equidad y calidad es que en el año 2005 nace la reforma de salud. En tres de los pilares de esta Reforma, ley 19.996, Régimen General de Garantías en Salud (GES) ${ }^{1}$, ley 19.937 de Autoridad Sanitaria ${ }^{2}$ y ley 20.584, ley de los Derechos y Deberes de los Pacientes ${ }^{3}$, se establece que la Calidad y Seguridad del Paciente es uno de los requisitos indispensables de la atención sanitaria. La Ley GES contempla la Calidad de la atención como una de sus garantías, estableciendo que los profesionales deben estar certificados en el registro de prestadores de la Superintendencia de Salud (SIS) a partir de julio de 2013 y que los prestadores institucionales deben estar acreditados a contar del 1 de julio de 2016 para atender las patologías GES.

Respecto a las especialidades odontológicas, es importante puntualizar que el Decreto $N^{\circ} 574$ de 2008 y posteriormente el Decreto $N^{\circ} 85$ de 2013 , establecen que dicha profesión cuenta con la especialidad de Cirugía y Traumatología Bucal y Máxilofacial (CBMF). Esta disciplina incluye el diagnóstico y tratamiento de enfermedades, lesiones y defectos que implican los aspectos funcionales y estéticos de los tejidos duros y blandos de la región oral y máxilofacial, así como el manejo oportuno y adecuado de cualquier complicación que de ello derive.

Esta especialidad se dicta en programas universitarios cuya duración es de tres a cuatro años con dedicación exclusiva. Dichos programas están implementados y desarrollados con los estándares de formación establecidos a nivel internacional para esta disciplina, velando por las competencias que sin duda cautelan el ámbito técnico y ético de dichos especialistas. Actualmente, existen 8 cursos de especialización en cirugía y traumatología bucal y máxilofacial: 5 en Santiago (Universidad de Chile, Universidad Mayor, Universidad de Los Andes, Universidad Andrés Bello y Pontificia Universidad Católica de Chile), 2 en Concepción (Universidad de Concepción y Universidad San Sebastián de Concepción) y 1 en Valparaíso (Universidad de Valparaíso).

Por otro lado, como establecen los Decretos $\mathrm{N}^{\circ} 57^{4}$ y $\mathrm{N}^{\circ} 8^{5}$ en su artículo segundo transitorio, también se reconocen como especialistas a aquellos profesionales certificados por la Corporación Nacional Autónoma de Certificación de Especialidades Odontológicas (CONACEO) así como aquellos que se hayan desempeñado como especialistas durante a lo menos cinco años en establecimientos asistenciales del Sistema Nacional de Servicios de Salud, en establecimientos dependientes de alguna de la instituciones pertenecientes a la Defensa Nacional, a Carabineros de Chile o a su Dirección de Previsión, o en el Servicio Médico Legal. El desempeño invocado por el profesional deberá ser certificado, según corresponda, por el Director del Servicio de Salud en el cual preste sus servicios, el Director de Sanidad del Ejército o la Armada, el Jefe de la División de Sanidad de la Fuerza Aérea, el Director de Salud o el Director de Previsión de Carabineros de Chile, o el Director del Servicio Médico Legal. Este régimen transitorio estaba establecido en el decreto $N^{\circ} 8^{5}$ hasta el año 2014, pero con el decreto $\mathrm{N}^{\circ} 65^{6}$ del Ministerio de Salud (MINSAL) y Ministerio de Educación (MINEDUC) fue prorrogado hasta el año 2019.

Bajo el argumento de la existencia de listas de espera y falta de especialistas en esta disciplina, el MINSAL decide crear un profesional cuya formación dure un año para que luego se desempeñe en la atención primaria de salud (APS), con este propósito establece la especialidad de Cirugía Bucal a través del Decreto $\mathrm{N}^{\circ} 65^{6}$ publicado el diario oficial el 13 de agosto de 2015 . Tenemos la convicción que con la creación de un profesional con menores competencias no se resguarda la calidad de los servicios sanitarios y no se da cumplimiento a la garantía de Calidad, exponiendo a los enfermos a eventos adversos de impredecibles desenlaces desde el punto de vista físico y psicológico, afectando muchas veces la calidad de vida de éstos o provocando un desenlace fatal.

Sumado a lo anterior, la cirugía bucal, como establece el Decreto $283^{7}$ de autoridad sanitaria, se ejecuta en un pabellón de cirugía menor, infraestructura inexistente en la gran mayoría de los centros de atención primaria, según consta en las respuestas a las consultas realizadas por transparencia a las diferentes Secretarias Regionales Ministeriales (SEREMI) del país, lo que compromete aún más la calidad y seguridad de esta atención.

Debido a que al momento del estudio no se cuenta con un registro del número de cirujanos y traumatólogos buco máxilofaciales formados en el país, se desconoce cuántos de ellos se desempeñan en los hospitales públicos y su distribución en los servicios de salud, así como también si se cumplen las condiciones de infraestructura necesarias para desarrollar la especialidad de acuerdo a la reglamentación vigente, hemos decidido efectuar un estudio a nivel de la red hospitalaria del sistema público nacional, con el objeto de efectuar un correcto diagnóstico desde el punto de vista del prestador individual e institucional que permita gestionar de manera adecuada las consultas y tratamientos de las patologías relacionadas con este ámbito de la atención sanitaria.

\section{MATERIALES Y MÉTODOS}

Se realizó un estudio descriptivo transversal para lo que se confeccionó un cuestionario dirigido a obtener datos sobre el prestador de salud individual e institucional, el que se aplicó en los 185 hospitales que constituyen la red pública nacional entre el mes de abril y junio de 2015.
El cuestionario fue aplicado telefónicamente sólo por la autora principal del trabajo, con objeto de evitar la variabilidad de las preguntas y controlar el sesgo del entrevistador. Se contactó a los jefes de los servicios odontológicos o en su defecto a los profesionales encargados de efectuar la cirugía de la especialidad en el recinto, considerando que eran las personas de mayor conocimiento en el tema a investigar.

El cuestionario se elaboró en base a preguntas objetivas, con una secuencia lógica. Cada pregunta midió sólo un objetivo y se proveyeron las definiciones legales de los recintos cuando fue pertinente.

Utilizando la calculadora de tamaño muestral de la SIS, se determinó que necesitábamos 20 centros hospitalarios para validar el instrumento. Estos se definieron aleatorizando mediante Randomizer los 185 hospitales de la red, numerándolos del 1 al 185, comenzando desde el norte con Arica y terminando en Punta Arenas. Se anotaron las observaciones de las preguntas en las cuales el profesional tuvo dificultad para contestar, con el objeto de poder efectuar las debidas correcciones

La encuesta consignó:

- Nombre y apellido del jefe de servicio odontológico, teléfono y correo electrónico.

- Nombre y apellido de profesional que ejecuta cirugías odontológicas y responde el cuestionario (cuando no se contactó al jefe de servicio odontológico).

- Acciones quirúrgicas que se realizan en el servicio odontológico.

- Nombre del o los profesional que realizan los procedimientos quirúrgicos y número de horas contratadas.

- Especialización de los profesionales. En caso de ser afirmatiitiva, el mecanismo de obtención de ésta.

- Lugar en que se realizan las acciones quirúrgicas: sala de procedimiento, pabellón de cirugía menor o pabellón de cirugía general.

El Decreto $283^{7}$ del MINSAL del año 1997 con sus modificaciones del 2005 y 2010 define Sala de Procedimiento como: Locales o recintos de establecimientos públicos o privados de salud destinados a efectuar procedimientos de salud, de diagnóstico o terapéuticos, en pacientes ambulatorios, y que no requieren de hospitalización. Estos deberán formar parte de un establecimiento de salud o se dependencia anexa a consultas de profesionales. Además, establece que en las salas de procedimientos se podrán realizar procedimientos de tipo invasivo y no invasivo. Se entenderá por procedimiento no invasivo, aquél que no involucra solución de continuidad de piel ni mucosas, ni acceso instrumental a cavidades o conductos naturales del organismo. Se entenderá por procedimiento invasivo aquél que involucra acceso instrumental a vías o conductos naturales del organismo y que requieren efectuarse con técnica aséptica, tales como radiografías con medio de contraste, endoscopías digestivas, respiratorias u otros procedimientos del área odontológica, tales como: exodoncia simple (diente erupcionado), biopsia de tejidos blandos o eliminación de bridas o frenillos

Respecto a la normativa de los Pabellones de Cirugía Menor, en su artículo 11 establece que son recintos destinados a realizar intervenciones quirúrgicas médicas u odontológicas, que no requieren la hospitalización del paciente, al que se le aplica sedación y/o anestesia local. Estos deberán formar parte de un establecimiento de salud o ser dependencia anexa a consultas de profesionales.

En su artículo 12 señala que se entenderá por Cirugía Menor a aquellos procedimientos invasivos que involucran solución de continuidad de piel o mucosa, acceso instrumental a cavidades naturales y que requieren ser realizados con técnicas estériles, tales como: procedimientos de cirugía plástica dermatología, oftalmología, serología, traumatología, ginecología, cirugía bucal, periodontal y otros.

Con el fin de conocer el número de profesionales formados a nivel país se tomó contacto con los encargados de los diferentes postítulos de las Universidades que lo imparten y con egresados de los programas que ya no se dictan, como es el caso de la Universidad de Antofagasta, obteniéndose el listado de todas las generaciones desde el inicio de cada uno de los programas

Todos los datos obtenidos en el trabajo fueron tabulados en planilla Excel.

\section{RESULTADOS}

Desde el punto de vista de la complejidad, los 29 Servicios de Salud existentes a lo largo de Chile, presentan una dotación heterogénea de establecimientos constituida por 185 hospitales, de los cuales 62 (33,5\%) corresponden a centros de alta complejidad, $24(13 \%)$ a mediana complejidad y $99(53,5 \%)$ a baja complejidad

Como se puede observar en la Tabla $\mathrm{N}^{\circ} 1$, en el extremo norte del país el servicio de salud Arica e Iquique cuenta cada uno de ellos sólo con un hospital de alta complejidad, por otro lado, el Servicio de Salud Arauco es el único que no posee este tipo de establecimiento. Hacia el sur aumentan los hospitales de baja complejidad y en la región metropolitana se concentran los establecimientos de alta complejidad.

La Tabla $\mathrm{N}^{\circ} 2$ nos permite identificar los centros que no otorgan prestaciones odontológicas y aquellos que no tienen especialistas en CBMF. Podemos advertir que de los 185 hospitales que conforman la red hospitalaria en $10(5,4 \%)$ no hay atención odontológica, de estos, $5(8 \%)$ son de alta complejidad, 2 (8\%) corresponden a mediana complejidad y $3(3 \%)$ a baja complejidad. Si a esto 
Tabla 1. Red Nacional de Salud Chile.

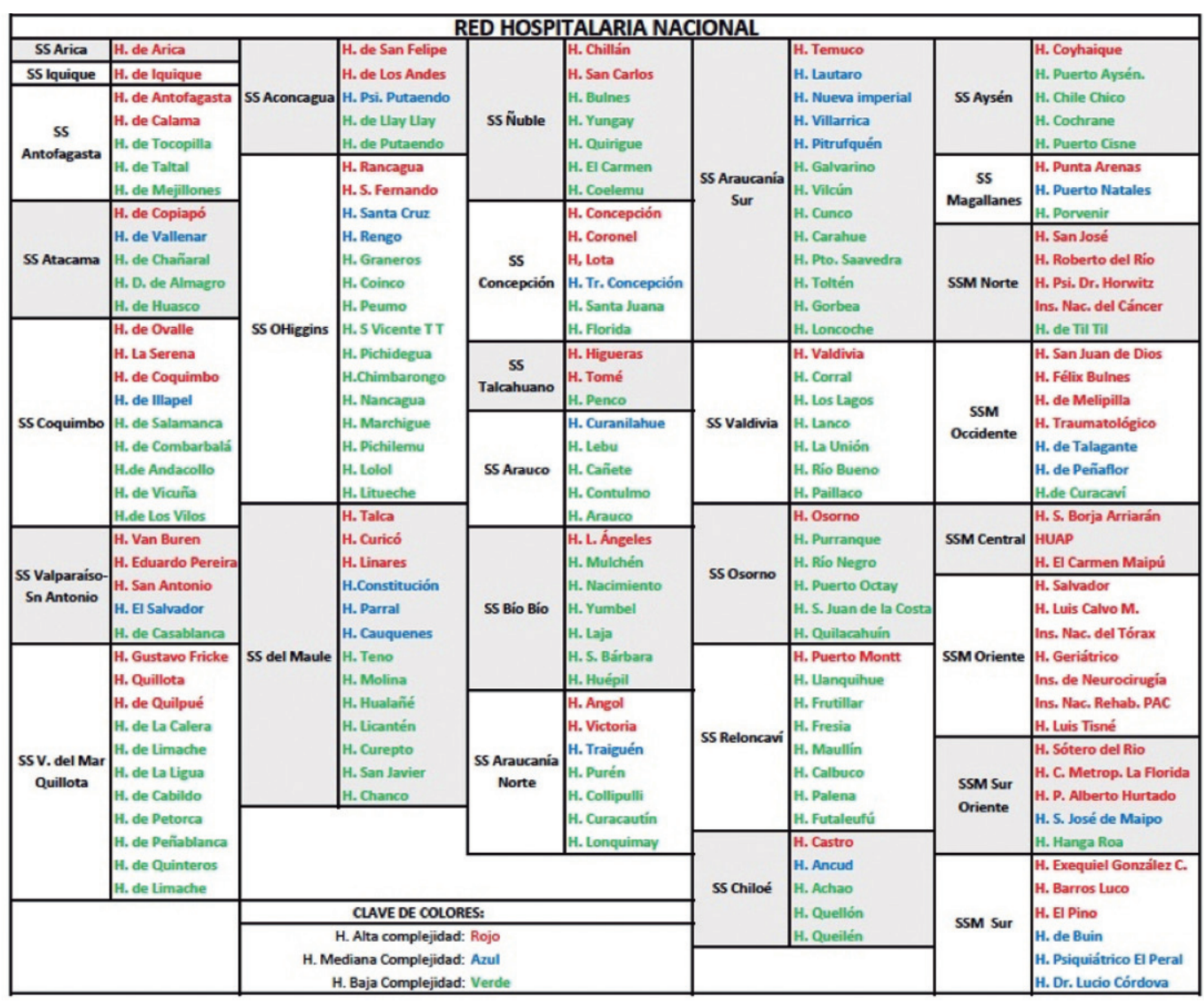

SS: Servicio de Salud.

le sumamos los hospitales en que a pesar de haber atención odontológica no hay cargos con profesionales preparados para resolver problemas quirúrgicos, la falta de resolutividad aumenta a $9(14,5 \%)$ recintos en los hospitales de alta complejidad, $11(45,8 \%)$ en los de mediana complejidad y $81(81,8 \%)$ en los de baja complejidad.

\section{Prestador individual}

En la Tabla $\mathrm{N}^{\circ} 3$ se puede apreciar cómo han ido surgiendo los programas de postítulos de la especialidad, siendo el más antiguo el impartido por la Universidad de Chile. En el año 2013 se implementan los postítulos de la Universidad de Los Andes y Andrés Bello y en el 2015 lo hace la Pontificia Universidad Católica, por lo que a la fecha del estudio no cuentan con egresados.

A junio de 2015 la suma de egresados de programas universitarios es de 257 profesionales, de los cuales 142 (55,3\%) se encuentran ocupando un cargo en los hospitales públicos. Existe un número de 78 alumnos cursando la especialidad, quienes han manifestado formalmente por escrito su disponibilidad para trabajar en el sistema público de salud, como consta en carta enviada por estos profesionales al MINSAL el 1 de junio del año 2015. Una vez egresados estos especialistas aumentarán en un $30 \%$ la dotación de cirujanos y traumatólogos buco máxilofaciales para el año 2018.

En la Tabla $\mathrm{N}^{\circ} 4$ se puede observar la cantidad de especialistas certificados por las diferentes vías establecidas que se desempeñan en los servicios de salud del territorio nacional.

De los 181 especialistas que trabajan en los hospitales públicos, 168 $(92,8 \%)$ lo hacen en los hospitales de alta complejidad y en $7(11,7 \%)$ de estos establecimientos cuentan con solo un profesional.

En $47(75,8 \%)$ de los 62 hospitales de alta complejidad de la red, la cirugía es efectuada por especialistas, en $6(9,7 \%)$ de ellos es efectuada por profesionales que no tienen la especialidad. En los 24 hospitales de mediana complejidad se desempeñan $8(4,4 \%)$ de los 181 especialistas y en $5(20,8 \%)$ recintos se encuentran solos, sin constituir equipos de trabajo.

En 6 (25\%) hospitales de mediana complejidad la cirugía es efectuada por especialistas y en $7(29,2 \%)$ por no especialistas.

En los 99 hospitales de baja complejidad ejercen $5(2,8 \%)$ de los 181 especialistas y los cinco trabajan solos en sus recintos. En $13(13,1 \%)$ de estos establecimientos la cirugía es efectuada por no especialistas.

Prestador Institucional

$\mathrm{Si}$ analizamos la infraestructura con que cuenta cada establecimiento para efectuar cirugía bucal y máxilofacial, encontramos que de los $53(85,5 \%)$ hospitales de alta complejidad donde se practica esta disciplina, en $46(74,2 \%)$ los especialistas tienen acceso a pabellón de cirugía mayor y en $9(14,5 \%)$ es la única posibilidad de ejecutar procedimientos quirúrgicos, sean estos de baja o alta complejidad. En $17(27,4 \%)$ de ellos tienen acceso a pabellones de cirugía menor y en $4(6,5 \%)$ es el único recinto quirúrgico existente. En 31 $(50 \%)$ hospitales de alta complejidad la cirugía bucal se realiza en salas de procedimiento y en $3(4,8 \%)$ de ellos es la única posibilidad para efectuar estas intervenciones, ya que no tienen acceso a pabellones.

En 22 de los 53 (41,5\%) establecimientos de alta complejidad que realizan cirugía bucal y máxilofacial se cumple con la norma 2837 de autoridad sanitaria para la ejecución de procedimientos quirúrgicos. En los $31(58,4 \%)$ recintos hospitalarios restantes no se cumple esta normativa (Gráfico $\mathrm{N}^{\circ} 1$ ).

De los 13 hospitales de mediana complejidad donde se realiza cirugía bucal y máxilofacial, solo en 6 de ellos los especialistas tienen acceso a pabellón de cirugía mayor. Por otro lado, en $3(23 \%)$ de los 13 establecimientos se cumple con la norma $283^{7}$ antes mencionada, en los $10(77 \%)$ recintos restantes no se cumple con esta normativa (Gráfico $\mathrm{N}^{\circ} 1$ ).

Del total de 18 hospitales de baja complejidad donde se realiza cirugía bucal y máxilofacial, en $6(33,3 \%)$ de ellos los especialistas tienen acceso a pabellón de cirugía mayor y en $4(22,2 \%)$ es la única posibilidad de ejecutar procedimientos quirúrgicos, sean estos de baja o alta complejidad. En $5(27,8 \%)$ de ellos tienen acceso a pabellones de cirugía menor y en $2(11,1 \%)$ es el único recinto quirúrgico. En $11(61,1 \%)$ hospitales de baja complejidad la cirugía bucal se realiza en salas de procedimiento y en $8(44,4 \%)$ de ellos es la única posibilidad para efectuar estas intervenciones.

En $7(38,8 \%)$ de 18 establecimientos de baja complejidad que realizan cirugía bucal y máxilofacial se cumple con la norma $283^{7}$ previamente citada. En los $11(61,1 \%)$ restantes no se cumple esta normativa (Gráfico №1).

Si analizamos la población por Servicio de Salud (SS), en el Gráfico №2 se puede apreciar que presenta una distribución normal, donde la mayor concentración se encuentra entre el SS Coquimbo (SSCoq) y el SS Concepción (SSCC). Hacia el sur se destaca el SS Araucanía Sur (SSAS) con una importante concentración de habitantes.

De acuerdo al número de horas de especialistas contratadas en los hospitales de la red pública, la media país corresponde a 22 horas de cirujano bucal y máxilofacial por cada 100.000 habitantes. Si observamos el Gráfico ํ3, podemos decir que a nivel país hay 2 SS que cumplen con este estándar, 13 SS están bajo este nivel con un rango que va de 11 a 21 horas por 100.000 habitantes y 13 lo hacen sobre este parámetro, entre 25 a 59 horas por 100.000 
Tabla 2. Hospitales con odontología sin especialistas y Hospitales sin odontología

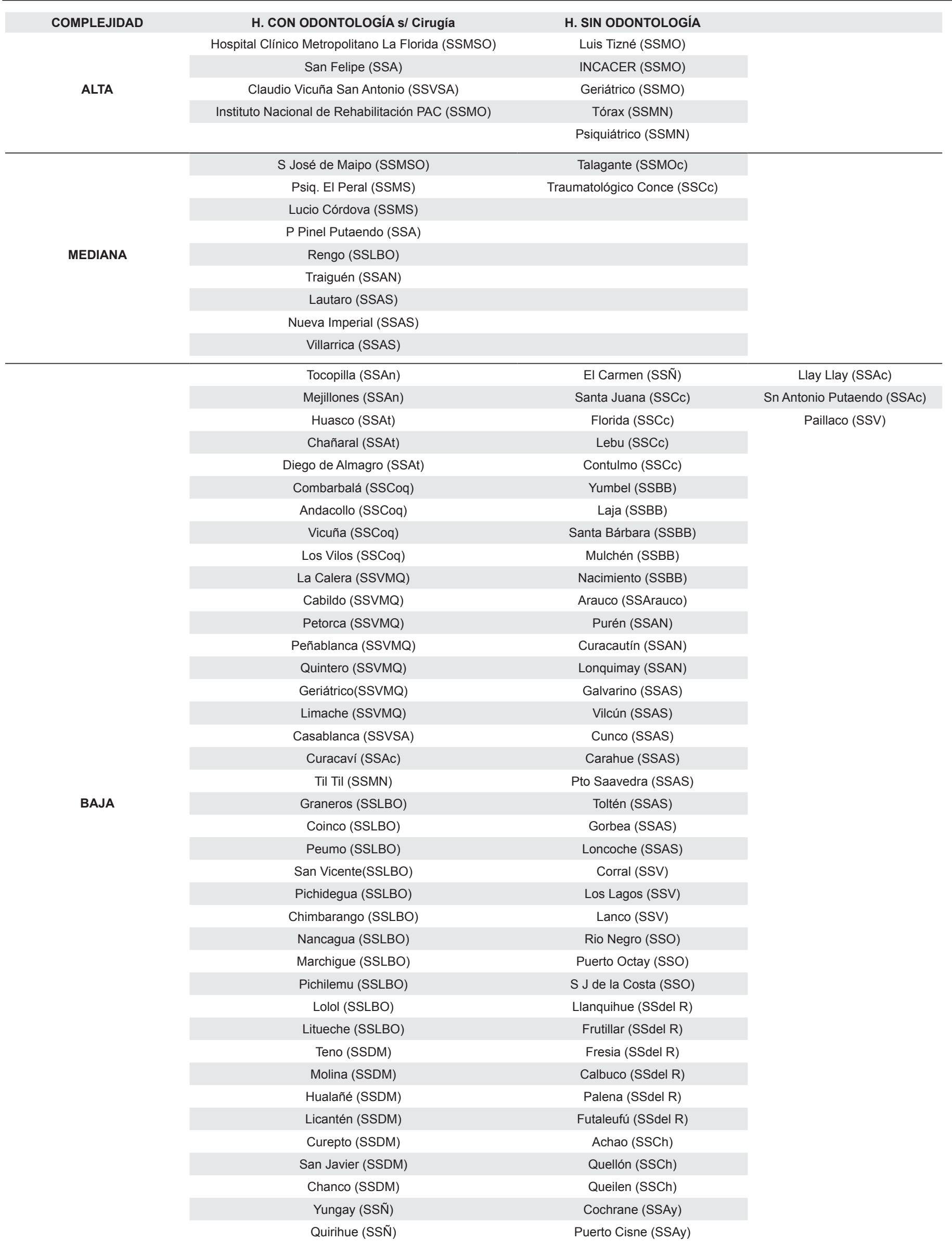


Tabla 3. Especialistas en CBMF y cargos en los SS.

\section{ESPECIALISTAS EN CBMF Y CARGOS EN LOS SS}

\begin{tabular}{|c|c|c|c|}
\hline Universidad & $\begin{array}{c}\mathbf{N}^{\circ} \\
\text { Especialistas }\end{array}$ & $\begin{array}{c}\% \\
\text { Especialistas }\end{array}$ & $\begin{array}{l}\text { Cargos } \\
\text { SS }\end{array}$ \\
\hline Chile (1978) & 135 & 53 & 59 \\
\hline Concepción (1993) & 28 & 11 & 18 \\
\hline Valparaíso (1997) & 64 & 25 & 46 \\
\hline Mayor (2008) & 16 & 6 & 9 \\
\hline Antofagasta (2007-2011) & 11 & 4 & 7 \\
\hline $\begin{array}{l}\text { Sn. Sebastián } \\
\text { Concepción }\end{array}$ & 3 & 1 & 3 \\
\hline TOTAL & 257 & 100 & 142 \\
\hline
\end{tabular}

CBMF: Cirugía y Traumatología Buco Máxilofacial. SS: Servicio de Salud

Tabla 4. Certificación de especialidad y cargos en los SS.

\begin{tabular}{|c|c|c|c|}
\hline \multicolumn{4}{|c|}{ CERTIFICACIÓN DE ESPECIALIDAD - CARGOS SS } \\
\hline ENTIDAD & $\begin{array}{c}\text { N}^{\circ} \text { CARGOS } \\
\text { SS }\end{array}$ & $\begin{array}{c}\text { \% CARGOS } \\
\text { SS }\end{array}$ & \\
\hline CONACEO & 18 & 10 & $\begin{array}{c}\text { Conocimiento y } \\
\text { experiencia }\end{array}$ \\
\hline DIRECTOR SS & 21 & 12 & $\begin{array}{l}\text { Constancia de } \\
\text { desempeño }\end{array}$ \\
\hline UNIVERSIDAD & 142 & 79 & $\begin{array}{l}\text { Egresado } \\
\text { de programa } \\
\text { universitario }\end{array}$ \\
\hline TOTAL & 181 & 100 & \\
\hline
\end{tabular}

CONACEO: Corporación Nacional de Certificación de Especialidades Odontológicas. SS: Servicio de Salud.

\section{Porcentaje de Cumplimiento Decreto 283 MINSAL}

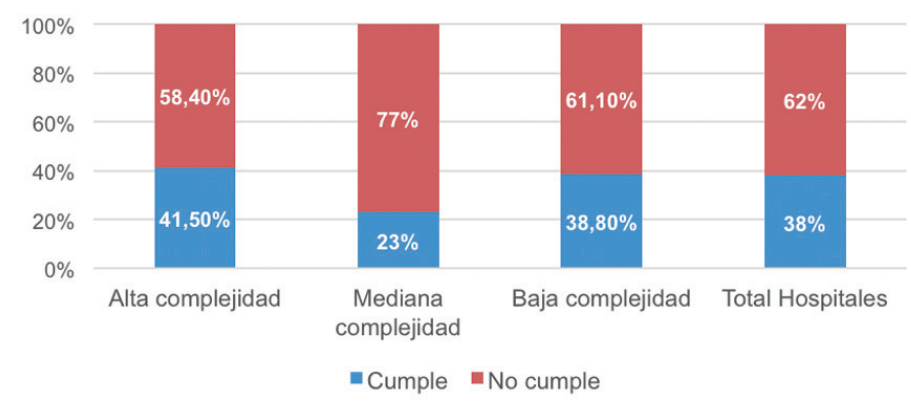

Gráfico 1. Porcentaje de Cumplimiento Decreto 283 MINSAL

\section{habitantes.}

Hay que destacar que $1 \mathrm{SS}$, Araucanía Norte (SSAN), no tiene horas de especialista y que 5 SS cuentan con una disponibilidad de 11 a 13 horas por 100.000 habitantes, es decir, menos de la mitad de las horas que se establecen como la media país.

Si se proyectan los Servicios con horas de CBMF bajo la media país sobre la distribución de la población por SS, podemos observar que estos corresponden en su mayoría a los de mayor concentración de habitantes, como se muestra en el Gráfico $\mathrm{N}^{\circ} 4$.

El Gráfico N ${ }^{\circ} 5$ muestra el detalle del déficit de horas de CBMF en los 14 SS que se encuentran bajo la media nacional.

\section{DISCUSIÓN}

Al analizar los resultados de esta investigación se puede inferir que la medida tomada por el MINSAL, de crear la especialidad de Cirugía Bucal claramente obedece a un error diagnóstico, originado por la falta de un estudio técnico respecto al recurso humano y la infraestructura con la que se cuenta para el desarrollo de esta especialidad a nivel país.
Los datos obtenidos en los postítulos de las Universidades demuestran que un $45 \%$ de los egresados no ha encontrado una posibilidad laboral en la red hospitalaria y que los 78 becados existentes a la fecha del estudio, aumentarán la dotación de especialistas en un $30 \%$ respecto a los existentes. Frente a estas cifras que demuestran la existencia de especialistas sin cargos en los hospitales públicos y una población de becados que seguramente no encontrará una fuente laboral en los Servicios de Salud, se torna difícil explicarle a la población por qué se decreta la creación de un nuevo especialista con menores competencias para atender a las personas que no tienen posibilidades de elegir su prestador de salud. Hoy día es de público conocimiento que muchos de los especialistas egresados de las becas para cierre de brechas costeadas por el MINSAL, no encuentran los recursos físicos en sus lugares de destinación para ejercer como tal, esto va desde el espacio para evaluar los pacientes, hasta un pabellón quirúrgico para solucionar diversas patologías, por lo que deben cumplir su jornada de trabajo realizando labores administrativas o relativas a un profesional sin especialización, provocando con esto la frustración del profesional, la pérdida de los recursos invertidos por el Estado en su formación y el menoscabo para la población que espera por ese especialista para solucionar de manera idónea su problema de salud.

Es relevante hacer notar que, en 17 hospitales de la red, $7(11,3 \%)$ de alta complejidad, $5(20,1 \%)$ de mediana y $5(5,1 \%)$ de baja complejidad, los profesionales se encuentran solos, sin constituir un equipo quirúrgico, lo que limita ostensiblemente su capacidad resolutiva.

Por otro lado, la cirugía bucal, como establece el decreto $283^{7}$ de autoridad sanitaria, se debe ejecutar en un pabellón de cirugía menor, infraestructura prácticamente inexistente en los centros de atención primaria, lo que compromete aún más la calidad y seguridad de la atención.

La ausencia de pabellones de cirugía menor en atención primaria no debería sorprender, ya que la evidencia encontrada en esta investigación nos demuestra que en numerosos hospitales de la red se adolece de esta infraestructura incluso en los centros de alta complejidad. En 9 de estos hospitales, el pabellón central es la única posibilidad de ejecutar procedimientos quirúrgicos sean simples o complejos, esto implica que cada vez que se presenta una urgencia médico quirúrgica la tabla odontológica es suspendida y postergada, aumentando las listas de espera. En los 31 (50\%) hospitales de alta complejidad donde la cirugía bucal se realiza en salas de procedimiento, es por la falta de un pabellón de cirugía menor. Es importante hacer notar que $8(12,9 \%)$ de estos hospitales están acreditados, a pesar que no cumplen la norma $283^{7}$ de autoridad sanitaria.

En $7(29,2 \%)$ hospitales de mediana complejidad no hay acceso a pabellón de cirugía mayor por lo que no se puede efectuar cirugía máxilofacial. En 10 $(41,7 \%)$ de estos establecimientos se usa sala de procedimiento para efectuar la cirugía bucal, incumpliendo la normativa vigente.

Del total de 99 hospitales de baja complejidad en sólo $18(18,2 \%)$ se efectúan procedimientos quirúrgicos, pero en $11(61,1 \%)$ de ellos no se cumple con el Decreto $283^{7}$.

Respecto a la cobertura de esta especialidad en el servicio público chileno, se ha podido establecer que corresponde a una media de 22 horas por 100.000 habitantes, es decir, 0,5 cirujanos orales y máxilofaciales a jornada completa por 100.000 habitantes. Sin embargo, no podemos plantear que esto es lo adecuado, ya que no tenemos certeza que en los servicios que están en esta cifra o sobre ella, no existan listas de espera. Perfectamente el servicio que tiene la mayor cantidad de horas por habitantes a nivel país, podría ser el estándar que se necesita a nivel nacional. Para tener un parámetro de comparación sobre este punto, se buscó información a nivel sudamericano, norteamericano y europeo; luego se buscó evidencia para realizar un análisis comparativo, utilizando como referencia los países de la Organización para la Colaboración y el Desarrollo Económico (OCDE), grupo al que Chile pertenece desde el año 2010.

El Gobierno de España en el año 2011 a través del Ministerio de Sanidad y Política Social e Igualdad, emitió un informe denominado: Ofertas y necesidades de especialistas médicos en España 2010-2025 por 100.000 habitantes la necesidad de cirujanos orales y máxilofaciales, esto es considerando que 1 equivale a un profesional con jornada completa ( 35 horas semanales).

Un informe del Royal College of Surgeons of England ${ }^{9}$ realizado el año 2011 en colaboración con las asociaciones de especialidades quirúrgicas, arroja la cifra de 1 cirujano oral y máxilofacial a jornada completa por 150.000 habitantes, lo que equivaldría 0.67 especialistas a jornada completa ( 35 horas semanales) por 100.000 habitantes.

También en el año $2011^{10}$, el Australian Institute of Health and Welfare presenta un estudio que reporta la existencia de 1 cirujano oral y máxilofacial a jornada completa (40 horas semanales) por 100.000 habitantes en ese país.

A nivel Sudamericano y Centroamericano no se encontró evidencia.

Si consideramos los resultados del Gráfico № 4, que reflejan el déficit de horas de CBMF a nivel país, es relevante tener presente al momento de planificar la cobertura de estas horas, la necesidad que estos profesionales trabajen en equipo con objeto de lograr una resolutividad adecuada, ya que los procedimientos quirúrgicos así lo requieren y además, se debe contemplar que el horario contratado resguarde una continuidad en la atención de los pacientes. 


\section{Población por Servicio de Salud}

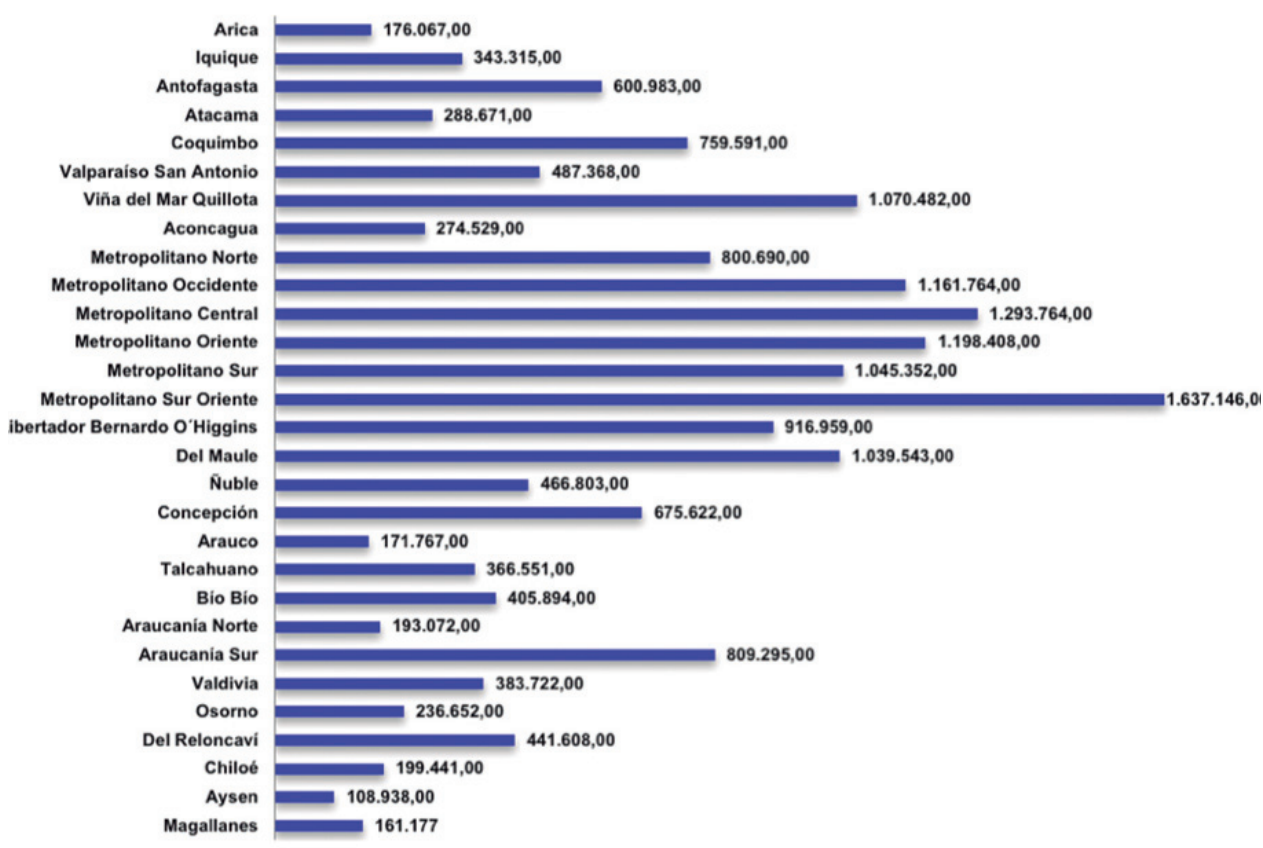

Gráfico 2. Distribución de población por Servicio de Salud en Chile

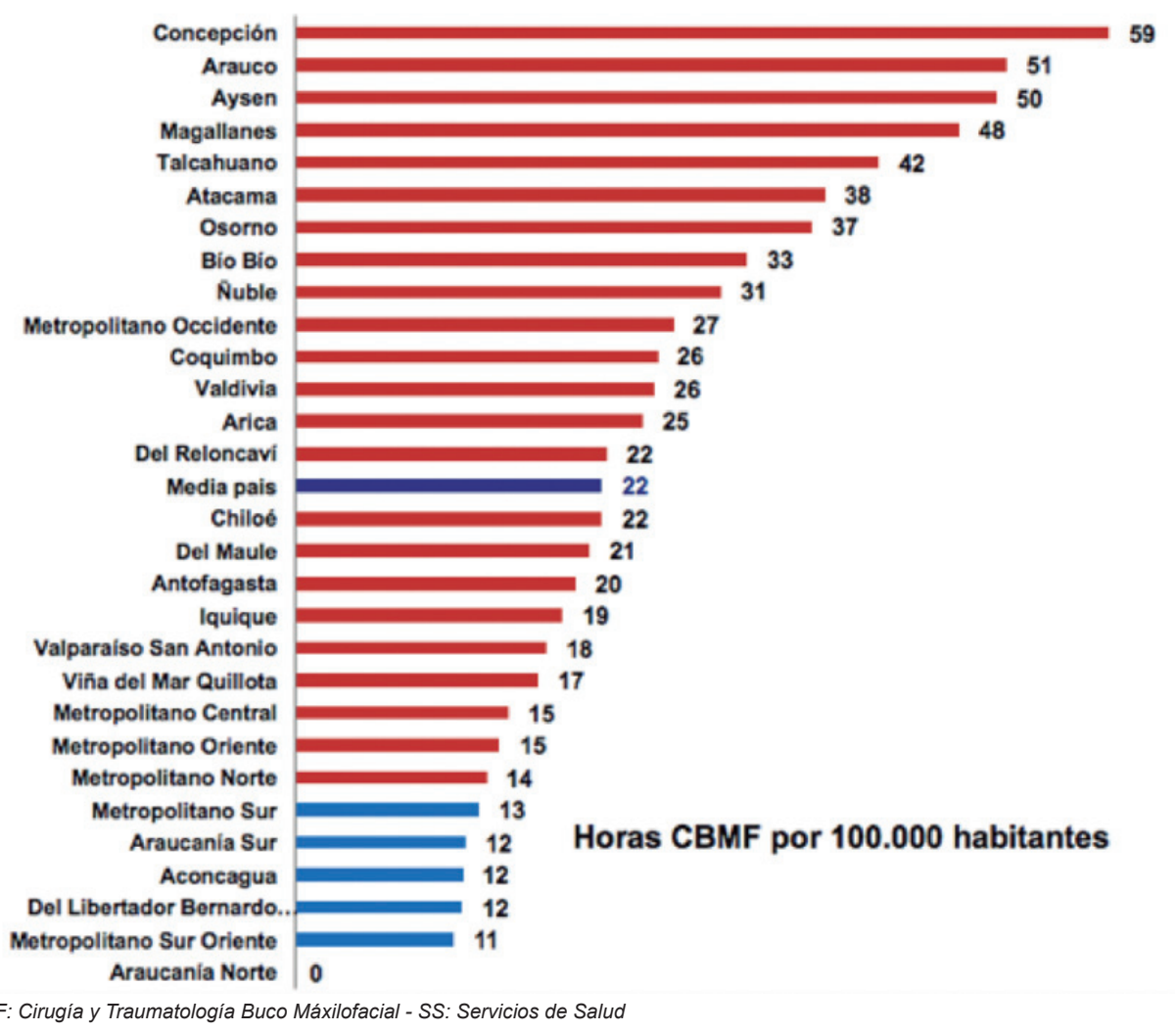

Gráfico 3. Media país en horas de CBMF por 100.000 habitantes, por SS.

\section{CONCLUSIONES}

Existe un error diagnóstico, originado por la falta de un estudio técnico respecto al recurso humano y la infraestructura con la que se cuenta para el desarrollo de la CBMF a nivel país.

En Chile hay especialistas en CBMF formados y en vías de formación, que han manifestado formalmente su aspiración de obtener un cargo en el servicio público, donde se desarrolla mayoritariamente la especialidad. Las cifras obtenidas permiten concluir que no hay déficit de especialistas.

Hay déficit de cargos de CBMF para constituir equipos quirúrgicos resolutivos en el sistema público y déficit de infraestructura adecuada, es decir faltan pabellones de cirugía menor y horas de acceso a pabellones de cirugía mayor para resolver las listas de espera de cirugía bucal y maxilofacial.

Frente a estos datos objetivos no hay razones técnicas que avalen la fragmentación de la especialidad de Cirugía y Traumatología Buco Máxilofacial, considerando que no es aceptable someter a riesgo de eventos adversos a los pacientes al ser atendidos por profesionales de menores competencias y en una infraestructura inadecuada. 


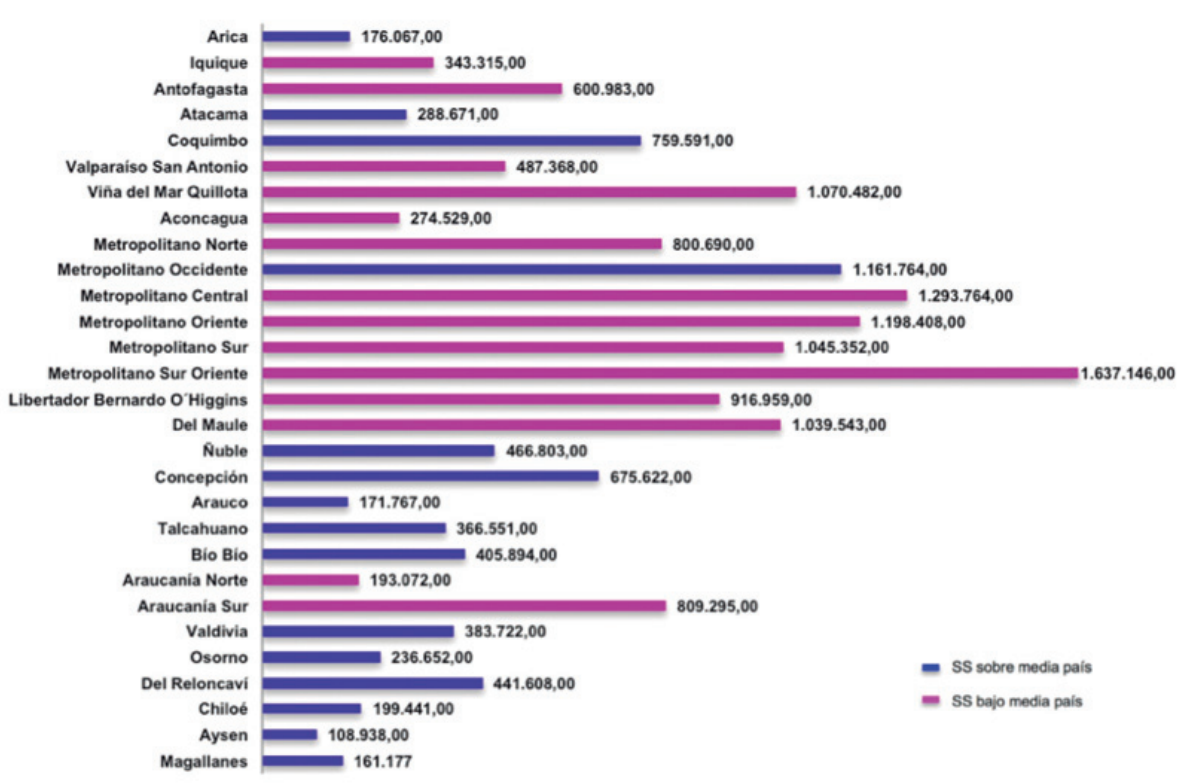

Gráfico 4. Distribución de horas de CBMF por SS y su población beneficiaria según media país.

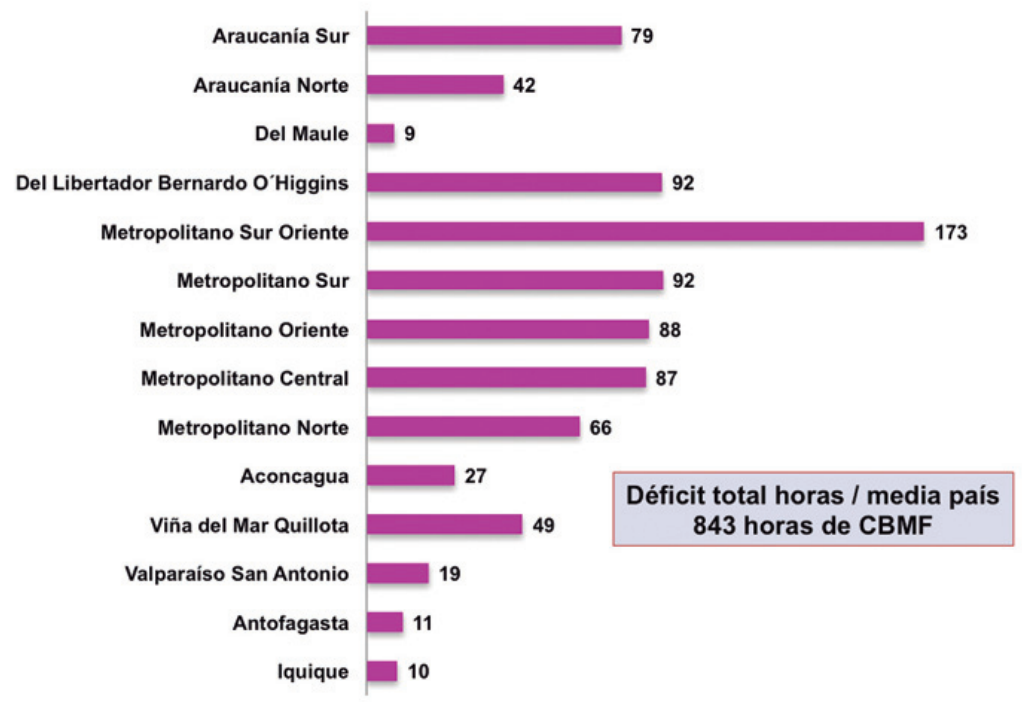

Gráfico 5. Déficit de horas CBMF/Media país

\section{AGRADECIMIENTOS}

Mis sinceros agradecimientos a todos los colegas que a lo largo de Chile aportaron esta valiosa información inédita en el país. Reciban mi admiración y respeto, sobre todo aquellos quienes en regiones remotas hacen patria ejerciendo nuestra noble profesión en el sistema público de salud.

Esperamos que estos datos sean un aporte para que al momento de confeccionar políticas públicas se resguarde, en todas sus dimensiones, la calidad y seguridad de la atención en este ámbito de la salud, tanto del usuario externo como interno.

\section{Bibliografía}

1. Ley Garantías Explícitas en Salud (GES). Decreto Supremo N ${ }^{\circ} 44$, de 2007, del Ministerio de Salud. Publicado en el Diario Oficial el 31 de enero de 2007.

2. Ley $N^{\circ} 19.937$ de Autoridad Sanitaria. Modifica el D.L. № 2763, de 1979. Ministerio de Salud. Publicado en el Diario Oficial el 24 de febrero de 2004.

3. Ley N ${ }^{\circ} 20.584$ de Derechos y Deberes de los pacientes. Ministerio de Salud. Publicado en el Diario Oficial el 24 de abril de 2012.

4. Reglamento de certificación de las especialidades y subespecialidades de los prestadores individuales de salud y de las entidades que las otorgan. Decreto 57 de 2007. Ministerio de Salud y Ministerio de Educación. Publicado en el Diario Oficial el 6 de noviembre de 2008. 5.Reglamento de certificación de las especialidades y subespecialidades de los prestadores individuales de salud y de las entidades que las otorgan. Decreto 8 de 2013. Ministerio de Salud y Ministerio de Educación. Publicado en el Diario Oficial el 1 de julio de 2013.

6. Reglamento de certificación de las especialidades y subespecialidades de los prestadores individuales de salud y de las entidades que las otorgan. Decreto 65 de mayo de 2015. Ministerio de Salud y Ministerio de Educación. Publicado en el Diario Oficial el 13 de agosto de 2015.
7. Decreto 283 de 1997. Ministerio de Salud. Publicado en el Diario Oficial el 12 de julio de 1997. Modificaciones: Decreto 141/05. Decreto227/05. Decreto 113/10.

8. Oferta y necesidades de especialistas médicos en España 2010-2025. (consultado 1 julio 2015) Disponible en:

http://www.msssi.gob.es/profesionales/formacion/necesidadEspecialistas/doc/11-Necesid adesMEspecialistas(2010-2025).pdf

9. Surgical Workforce 2011. A report from The Royal College of Surgeons of England in collaboration with the surgical specialty associations. (consultado 7 julio 2015) Disponible en:

https://www.rcseng.ac.uk/library-and-publications/college-publications/docs/surgicalworkforce-report-2011/

10. Australian Institute of Health and Welfare 2013. Dental workforce 2011. National health workforce series no. 4. Cat. no. HWL 50. Canberra: AlHW. 